\title{
Parosteal osteosarcoma: a diagnostic dilemma on non-healing following tooth extractions: a case report
}

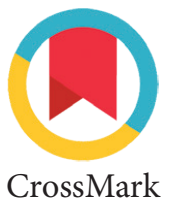

\author{
Rahma Y. Damanhuri", Prihartiningsih, Poerwati S. Rahajoe
}

\section{Abstract}

Objective: This paper reports a case of mandibular ramus parosteal osteosarcoma, discussing diagnostic problems and reviewing treatment variations.

Methods: A 37 year old male patient was referred by a private hospital with a history of abscesses treatment and removal of his right lower molar 1.5 years ago. The wound in his tooth socket was not healed. Swelling with abscesses recurred and was diagnosed as osteomyelitis. The Orthopantomogram (OPG) result showed a description of osteomyelitis, and the Multi Slice Computed Tomography (MSCT)
3D confirmed the suspected osteosarcoma. Histopathologic results indicated parosteal osteosarcoma.

Result: Hemimandibulectomy and chemotherapy were performed with good results.

Conclusion: This parosteal osteosarcoma was difficult to diagnosis quickly because the initial image resembled osteomyelitis but it was low grade and rare; therefore, meticulous and accurate diagnosis was deemed necessary. Chemotherapy-combined surgery was thereby performed for treating this parosteal osteosarcoma.
Department of Oral and Maxillofacial Surgery, Faculty of Dentistry, Gadjah Mada University, Yogyakarta, Indonesia
*Corresponding to:

Rahma Y. Damanhuri, Department of Oral and Maxillofacial Surgery, Faculty of Dentistry, Gadjah Mada University, Yogyakarta, Indonesia rahma.yulinda.d@gmail.com

Received: 4 January 2018 Revised: 11 February 2018 Accepted: 19 February 2018 Available online: 1 April 2018

Keywords: Parosteal osteosarcoma, Low-grade osteosarcoma, 0steomyelitis, Hemimandibulectomy

Cite this Article: Damanhuri RY, Prihartiningsih, Rahajoe PS. 2018. Parosteal osteosarcoma: a diagnostic dilemma on non-healing following tooth extractions: a case report. Journal of Dentomaxillofacial Science 3(1): 50-53. D0I: 10.15562/jdmfs.v3i1.698

\section{Introduction}

Osteosarcoma is a malignant neoplasm that commonly occurs in the long bones. Its predilection refers to distal femoral metaphysis, proximal tibia, and humeral metaphysis while the predilection on the mandible, maxilla, rib, vertebra, hand and foot bone is rare. ${ }^{1,2}$

Osteosarcoma arises from an osteogenic matrix ${ }^{3}$ derived from the mesenchymal cells ${ }^{4,5}$ with the presence of osteoid-forming atypical cells within the protein matrix produced by bone cells. ${ }^{1}$ Osteosarcoma is rare in the head and neck region and mostly occurs in decade 3 and 4.,4,6 The incidence of osteosarcoma in the jaw is only $5-6.5 \%$ of all osteosarcoma incidences. $^{1,7}$ Its occurrence to the mandible and the maxilla is almost at the same frequency. In the mandible, it often occurs in the posterior body and ramus whereas, in the maxilla, it commonly occurs in the alveolar ridge region, sinus and palatum base. ${ }^{1,5,6}$

Parosteal osteosarcoma belongs to the juxtacortical type of osteosarcoma. When first described by Geschickter and Copeland in 1951, this osteosarcoma was benign and gradually grew to be malignant but rarely occurred in the jaw. In 1961, Som and Peimer first described parosteal osteosarcoma in the jaw and reported only 12 cases in the literature. $^{8}$

Parosteal osteosarcoma is often misdiagnosed as a benign fibrous lesion while it is generally fibrous dysplasia. ${ }^{2,8}$ Low-grade osteosarcoma in conventional radiographic examination (periapical or panoramic) has a sclerotic appearance in the tooth apex that resembles an inflammatory periapical lesion. $^{2}$ As a result, a number of clinicians misdiagnose the case, resulting in a lower survival rate of the patients.

The management of parosteal osteosarcoma varies from resection surgery combined with chemotherapy, ${ }^{1}$ complete and extensive excision, ${ }^{9,10}$ and postoperative adjuvant chemotherapy. ${ }^{4}$

This paper reports a case of parosteal osteosarcoma of the right mandibular ramus as well as discusses difficulties in diagnosing the case and reviews possible treatment variations.

\section{Case Report}

A 37 year old male patient was referred by a private hospital in Central Java, Indonesia to the Department of Oral Surgery Dr Sarjito Hospital Yogyakarta. Approximately 1.5 years prior, he was treated for abscesses and the removal of his tooth 46-48 allegedly causing a focal infection. However, the wound in his tooth socket was not well healed as there was an open bone. Swelling with abscesses recurred about 1 month prior to hospital admission and was diagnosed with osteomyelitis. 

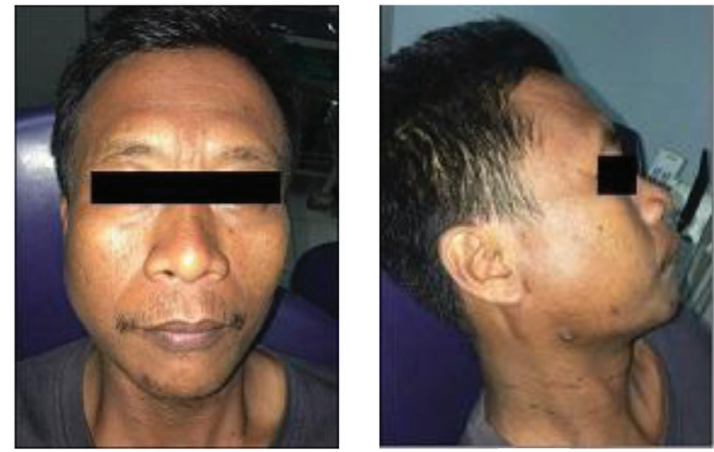

A

Figure 1 Clinical features: A. Extraoral front view, B. Extraoral side view, C. Intraoral

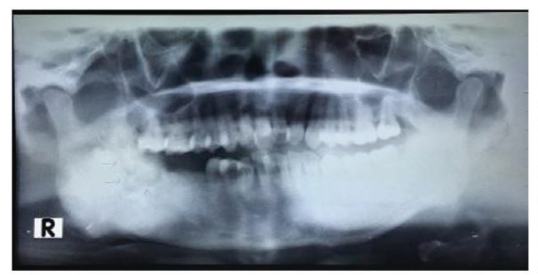

A

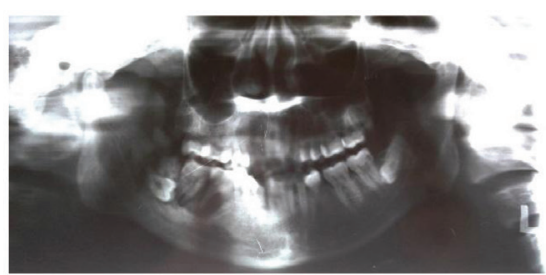

B
Figure 2 OPG Results: A. Obtained in 2014, B. Obtained in 2016

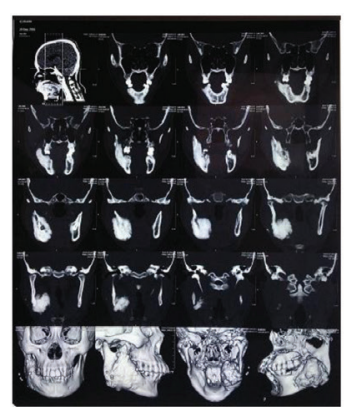

A

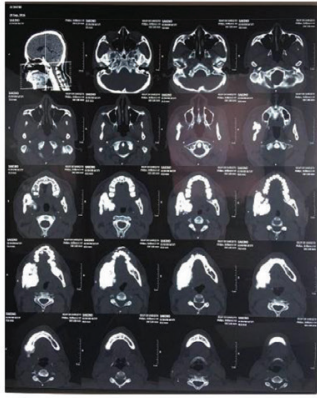

B

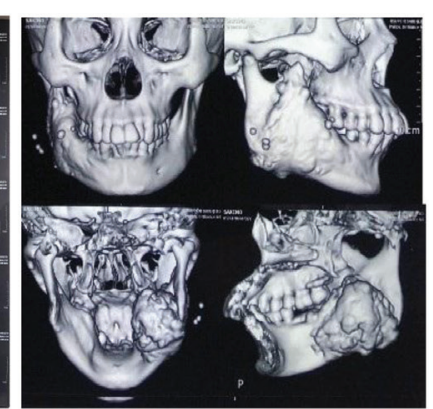

C
Figure 3 A. MSCT axial view, B. MSCT coronal view, C. 3D

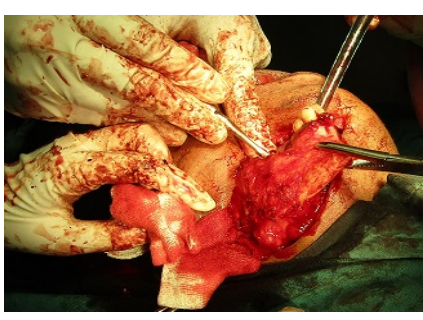

A

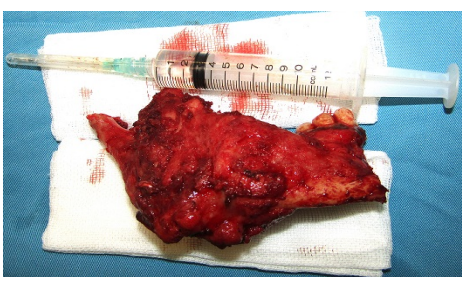

C

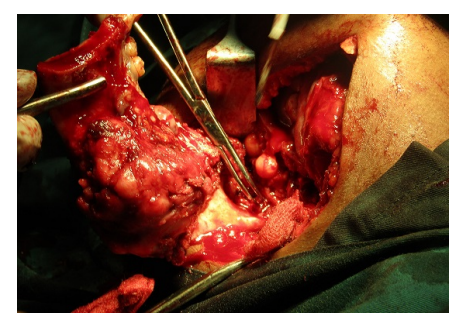

B

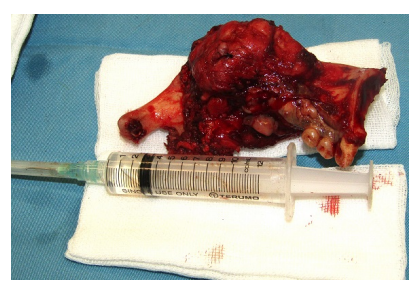

D
Figure 4 Hemimandibulectomy: A and C. Buccal view, B and D. Lingual view
The extraoral clinical examination found an asymmetrical face with a mild swelling on the right cheek to the right mandibular angulus figure $1 \mathrm{~A}$. An incision scar was also found on the right submandible but there was no pus figure 1B. The intraoral clinical examination observed normal opening of the mouth, open bones in the posterior right mandible in region 47-48, lingual and buccal swelling in region 46-48, no pus, edentulous ridge of tooth region 46-48, reddish gingiva region 44-48 in an irregular shape, pain when suppressed, no paresthesia, poor oral hygiene with calculus, and stain on the upper and lower jaws figure 1C. Nothing was palpable by the lymph node examination. Meanwhile, a hematologic examination found no abnormalities.

The OPG result in 2014 showed a radiolucent area in the apex of tooth 46-47 with a well-defined border, embedded tooth 48 , and a slightly non-specific sclerotic appearance in superior tooth 48 figure 2A. The OPG performed at Dr Sardjito Hospital in 2016 resulted in a sclerotic appearance of the tooth 46-48 region with an angulus, irregular shape and a borderless, missing tooth 46-48 figure 2B.

The 3D MSCT indicated soft tissue swelling of the extracranial right mandibular region, hard tissue tumours in the right mandibular angulus to the corpus, expanding to the buccal, lingual and anterior of tooth 44 , the periosteal reaction $(+)$ of the sunray type, and osteoid calcification (white arrow). The 3D MSCT results were suspected as osteosarcoma figure 3 .

Thorax X-ray examination, abdominal ultrasonography (USG) (liver, vesica felea, lien, pancreas, kidneys, vesica urinaria and prostate) and a bone survey showed no signs of metastasis. The parosteal osteosarcoma stage of the right mandible belongs to stage IA (G1S1M0) based on the enneking staging.

A wide excision hemimandibulectomy was performed by cutting the symphysis region. The lesions appeared as nodules of non-stalked lobes attached firmly to the cortex, hard when palpated, enlarging to the buccal and lingual with an anterior border to the tooth 43 region and posterior to $3 \mathrm{~mm}$ inferior of the condyle condensing neck figure 4 . Histopathologically, it resulted in parosteal osteosarcoma (PA Result: JRS-16-3673)

Postoperative control on day 10 showed surgery wound healing, reddish in colour, and well-attached simple interrupted sutures which were then removed. The patient was subsequently referred to the oncology department for continued treatment and planned chemotherapy. The 16th day postoperative control indicated colour and texture of the surgical wound was the same as the surrounding tissue, this suggested good wound healing figure 5 . The patient is currently undergoing the chemotherapy treatment and is in a good general condition. 

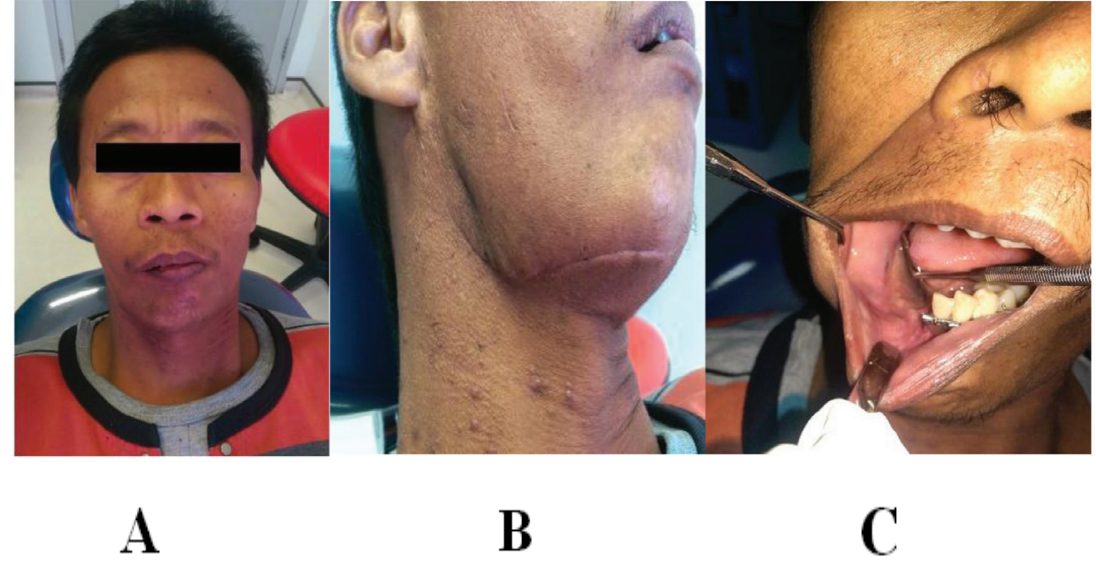

Figure 5 Clinical photos 16 days after hemimandibulectomy, A. Extraoral front view, B. Extraoral side view, C. Intraoral

\section{Discussion}

Parosteal osteosarcoma is one of the rare types of malignant neoplasms. In this case, it was initially suspected as osteomyelitis because of the history of abscess inducing infection. Experts claimed that the infection was associated with the occurrence of osteosarcoma. ${ }^{5,6}$ Several cases of osteosarcoma with abscesses have been reported..$^{5,11}$

Osteomyelitis is an inflammation not only involving the medulla portion of bone but also involves the cortical and periosteum. Clinical features of osteomyelitis are usually limited in the presence of minimal pain, non-healing of the bone and over soft tissue lesions with induration, intraoral or extraoral fistulas. Features also include bone elasticity, enlargement of the mandible due to new bone deposition in the subperiosteal region, and the teeth in the affected area tend to be unsteady and sensitive to palpation and percussion. ${ }^{12}$

The presence of a non-healing wound in this case also needs to be suspected because this tendency is common in osteosarcoma. ${ }^{6}$ There has been widely reported cases of a non-healing socket after tooth extraction that turned out to be cancer such as squamous cell carcinoma, adenocarcinoma, intraosseous carcinoma (PIOC), mandibular osteosarcoma, and Ewing's sarcoma. ${ }^{13-17}$ Differential diagnosis of osteosarcoma of the jaw includes osteoma, torus, chondrosarcoma, Ewing's sarcoma, bone metastases, fibrous dysplasia, osteomyelitis, and other lesions, such as fibrosarcoma, leiomyosarcoma, and rhabdomyosarcoma. ${ }^{6}$

Parosteal osteosarcoma belongs to a juxtacortical osteosarcoma. Osteosarcoma is generally divided into 3 groups: conventional type, which appears in the medullary cavity (intracortical), juxtacortical osteosarcoma derived from periosteal surface clinicopathologically categorized into 2 groups comprising parosteal osteosarcoma and periosteal osteosarcoma, and extraskeletal osteosarcoma (extra-osseus). ${ }^{1,3}$

Osteosarcoma is difficult to distinguish from osteomyelitis by the conventional radiography. ${ }^{18}$ The radiographic similarity is associated with increased bone sclerosis. The destruction of the bone cortical border is a typical characteristic of a malignancy but in the osteomyelitis, cortical destruction is not different. Osteomyelitis is often unrelated to the presence of tooth movement involved whereas, it is common in osteogenic sarcomas. The radiographic form of osteosarcoma of the jaw may be lytic, sclerotic and mixed, but it histopathologically has nothing to do with the osteosarcoma type. ${ }^{19}$

In this case, a sunray-type periosteal reaction was seen in the MSCT examination but invisible in the OPG. In conventional radiographs, the periosteal reaction of the mandibular bone is often seen through occlusal $\mathrm{x}$-ray examination because it may exhibit bicortical expansion. ${ }^{6,19}$ The typical radiographic appearance of osteosarcoma, in this case, was sunray-type periosteal irregular bone form, bone destruction and wide transition zone. . $, 7,20$, , 12

\section{Conclusion}

Oral parosteal osteosarcoma is a malignancy that is difficult to diagnose quickly because its initial lesions mostly resemble other lesions. In this case, the initial appearance resembles osteomyelitis. Wide excision hemimandibulectomy was performed and continued with chemotherapy to give good results.

\section{Acknowledgment}

The government of the study country and the World Health Organization did not influence this analysis nor the decision to publish these findings. The study did not receive any financial support

\section{Conflict of Interest}

The author report no conflict of interest.

\section{References}

1. Balwani SR, Tupkari JV, Barpande S. Parosteal osteosarkoma of the mandibula. J Oral Maxillofacial Pathol 2006;10: 10-14.

2. Diniz AF, Filho JAA, Alencer RC, et al. Low-grade central osteosarcoma of the mandible: a case study report. Oral Surg, Oral Med, Oral Pathol, Oral Radiol and Endodontol 2007;103: 246-252.

3. Nanci NF, Marchiori E, Vianna AD, et al. Osteossarcoma parosteal: aspectos na radiologia convencional TT-Parosteal osteosarcoma: conventional radiology findings. Radiol Bras 2007;40: 81-86. 
4. Chakravarthi PS, Vivekanand S, Kattimani VS, et al. Juxtacortical osteosarcoma of the mandible: challenges in diagnosis and management. National J Maxillofac Surg 2015;6: 127-131.

5. Kaur H, Singh A. Osteosarcoma of jaw-Case report and review of literature. IJMDS 2015;4: 653-657.

6. Bojan A, Christy W, Chanmougananda S, et al. Osteosarcoma of mandible : a case report and review of literature. J Clin Diagnostic Res 2012;6: 753-757.

7. Bhatia V, Sharma S, Sood RG, et al. Bony beard: osteosarcoma of the mandible with exuberant sunburst periosteal reaction. Int J Med Biomed Res 2012;1: 85-88.8. Huang TC, Monsour PA, Chahoud CD. Parosteal osteosarcoma: report of a case and review of the literature. Aust Dent J 2010;55: 86-91.

9. Hang JF, Chen PCH. Parosteal osteosarcoma. Arch Pathol Lab Med 2014;138: 694-699.

10. Singh S, Sangwan M, Verma R, et al. Case report: parosteal osteosarcoma of wrist: a rare case report. Int J Healthcare \& Biomed Res 2014;2: 60-62.

11. Anil S, Krishnan AP, Rajendran R. Osteosarcoma of the mandible masquerading as a dental abscess: report of a case. Case Rep Dent 2012;1: 1-5.

12. Malik NA. Textbook of oral and maxillofacial surgery, 3rd ed. New delhi: Jaype Brother Medical Publisher; 2012. p. 713-740.

13. Henry CJ, Stassen LF. The non-healing extraction socket: a diagnostic dilemma-case report and discussion. Irish Dental Assocation (IDA) 2016;62: 215-220.

14. Bansal D, Grewal HK, Jawanda M, et al. A rare site of adenocarcinoma not otherwise specified: a case report. Indian J Stomatol 2012;3: 282-285.
15. Lakshman AR, Kanneppady SK. An unusual case of primary intraosseous carcinoma of the mandible. J Dentofac Sci 2013;2: 5-8.

16. Kalburge JV, Sunil K, Sahuji SK, et al. Osteosarcoma of mandible. J Clin Diagnostic Res 2012;6: 1597-1599.

17. Lokesh K, Kannabiran J, Eregowda S. Maxillary ewing's sarcoma: a report of innocuous lesion with nocuous pathology. J Indian Academy Oral Med \& Radiol 2016;28: 203-206.

18. Petrikowski CG, Pharoah MJ, Lee L, et al. Radiographic differentiation of osteogenic sarcoma, osteomyelitis and fibrous dysplasia of the jaws. Oral Surg, Oral Med, Oral Pathol and Oral Radiol 1995;80: 744-750.

19. Khorate MM, Goel S, Singh MP, et al. Osteosarcoma of mandible: a case report and review of literature. J Cancer Sci \& Therapy 2010;2: 122-125.

20. Athar M. Malignant mandibular tumors : two case reports of rare mandibular tumors in a single institution. Arch Med Biomed Res 2014;1: 22-29.

21. Ismardianita E, Nasrul E, Yanwirasti, et al. Effect of ethanol extract of myrmecodia pendens on TGF- $\beta 1$ expression and osteoblast cells after tooth extraction (experimental research on cavia cobaya). J Dentomaxillofac Sci 2017;2: $150-154$.

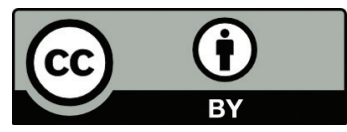

This work is licensed under a Creative Commons Attribution 